\title{
Unruptured aneurysms in the elderly: handle with care
}

\author{
Tarek Abuelem, MD, ${ }^{1}$ David Dornbos III, MD, ${ }^{2}$ and Adam Arthur, MD, MPH ${ }^{1}$ \\ ${ }^{1}$ Department of Neurosurgery, University of Tennessee Health Sciences Center and Semmes-Murphey Clinic, Memphis, \\ Tennessee; and 'Department of Neurosurgery, The Ohio State University Wexner Medical Center, Columbus, Ohio
}

$\mathrm{M}$ OST cerebral aneurysms never rupture, but when they do, it is often a calamity for the unfortunate patient. Whereas the decision whether or not to treat a ruptured aneurysm is relatively straightforward, the treatment algorithm for incidentally discovered unruptured aneurysms is considerably more difficult. Given the increased sensitivity and utilization of imaging modalities, these aneurysms are being detected more often. Because medical complaints are more common in the growing elderly population, medical imaging is even more frequently used for these patients. As with all patients, in the elderly the treatment to eliminate the risk of aneurysmal rupture must be weighed against the risk associated with intervention. The elderly population carries inherently elevated surgical risks given their age, increased comorbidities, and slower recovery. Furthermore, treatment benefit is relatively lessened in this population, because the total risk of rupture is limited by the remaining life expectancy.

This study assesses perioperative outcomes and provides cost analyses of treatments offered to elderly patients with unruptured cerebral aneurysms. ${ }^{12}$ An age of $\geq 65$ years was used to define the study population, which is a generally accepted age cutoff. Although chronological age is an important indicator of general health and life expectancy, the variability of health in advancing age limits its value when used as the sole indicator of fitness and physiological reserve. ${ }^{3,6}$ In addition to characterizing perioperative outcomes, this study provides additional insight into the increasing rate of interventions for unruptured cerebral aneurysms, which has a substantial impact on health care spending, necessitating quantification of the associated costs.

The Nationwide Inpatient Sample provides crosssectional discharge data from approximately $20 \%$ of all hospitals in the US, lending a representative sample with good external validity. Data extracted to define mortality and cost are more reliable than data describing periopera- tive morbidities, given the inherent flaws of the retrospective design. The authors accurately refer to a cost-analysis rather than a cost-effectiveness study, because long-term follow-up is missing. Ultimately, the cost of any treatment modality involves subsequent imaging and future treatment costs, which were not evaluated in this study. Although previous studies have suggested more favorable outcomes and lower mortality rates with aneurysm coiling compared to surgical clipping, this paper was not designed (nor was it statistically powered) to address this question..$^{8-10}$ It does, however, provide useful statistical information comparing these two treatment modalities.

Following microsurgical clipping, mortality in the elderly cohort was found to be $2.2 \%$, as opposed to $0.8 \%$ mortality in nonelderly patients, a difference that was found to be statistically significant. Interestingly, there was no difference in mortality following endovascular treatment, with rates of $0.9 \%$ and $0.6 \%$ in the elderly and nonelderly populations, respectively. It is important to note that this finding is a comparison of the mortality rate between age groups associated with a specific treatment modality, but does not directly compare mortality in the elderly patients based on treatment modality. These findings imply that the interaction of age and complications probably plays a more significant role in the elderly population following more invasive open microsurgery.

As part of the aging process, the cerebral vasculature undergoes anatomical and physiological changes. Anatomically, blood vessels increase in tortuosity with age and undergo changes in wall thickness and strength. This results in new hemodynamic stresses, which favor aneurysm formation. These new aneurysms have wall characteristics that differ from aneurysms arising in the younger population. It remains unclear whether the genesis of certain de novo aneurysms in the elderly has the same etiology as de novo aneurysms in the younger population, and therefore, the natural history of aneurysms may differ depend- 
ing on patient age. Unfortunately, natural history studies have not made a distinction regarding the risk of aneurysm rupture based on the age at which the aneurysm was discovered. Simply extrapolating current natural history data to newly discovered aneurysms in the elderly may over- or underestimate the risk of rupture. Physiologically, the autoregulatory capacity and blood flow in the "aging" cerebral vasculature are not well understood, but may result in less protection from the systemic effects of hypertension. ${ }^{4,7}$

These age-related physiological changes also affect the overall morbidity and mortality outcomes of both surgical clipping and endovascular coiling. With open microsurgery, the risk of stroke secondary to definitive and/or temporary clipping increases due to the possibility of plaque rupture, vessel wall rupture, and decreased compensatory autoregulation..$^{5}$ Increased vascular tortuosity increases the difficulty in endovascular access and subsequent treatment as well.

Due to these anatomical and physiological changes, the uncertain natural history of unruptured aneurysms in the elderly, and the higher rate of comorbidities, a conservative approach should be the first consideration in the decision making process. Ample clinical experience and an advanced set of operative skills are clearly required to care for this patient population. Complication rates cited in the current literature probably underestimate the risks of adverse sequelae following treatment, and do not accurately inform the patient or provider about the actual risks related to treatment of unruptured intracranial aneurysms in the elderly population, given the impact of pathophysiological variances, age, and comorbidities not present in the general population. Further studies evaluating the natural history and long-term outcomes following treatment or conservative management of aneurysms in the elderly population will help to determine the optimal treatment modality for these patients. Similar to the quality-of-care requirements set for carotid endarterectomy ${ }^{2,11}$ and stenting, ${ }^{1}$ the treatment of unruptured cerebral aneurysms should be handled at centers of excellence in both open and endovascular surgery, especially for elderly patients with unruptured aneurysms.

https://thejns.org/doi/abs/10.3171/2018.2.FOCUS1871

\section{References}

1. AbuRahma AF, Campbell JE, Hariri N, AbuRahma J, Dean
LS, Bates MC, et al: Clinical outcome of carotid artery stenting according to provider specialty and volume. Ann Vasc Surg 44:361-367, 2017

2. AbuRahma AF, Stone PA, Srivastava M, Hass SM, Mousa AY, Dean LS, et al: The effect of surgeon's specialty and volume on the perioperative outcome of carotid endarterectomy. J Vasc Surg 58:666-672, 2013

3. Artz AS: Biologic vs physiologic age in the transplant candidate. Hematology Am Soc Hematol Educ Program 2016:99-105, 2016

4. Buijs PC, Krabbe-Hartkamp MJ, Bakker C, de Lange EE, Ramos L, Breteler M, et al: Effect of age on cerebral blood flow: measurement with ungated two-dimensional phasecontrast MR angiography in 250 adults. Radiology 209:667674, 1998

5. Byoun HS, Bang JS, Oh CW, Kwon OK, Hwang G, Han JH, et al: The incidence of and risk factors for ischemic complications after microsurgical clipping of unruptured middle cerebral artery aneurysms and the efficacy of intraoperative monitoring of somatosensory evoked potentials: a retrospective study. Clin Neurol Neurosurg 151:128-135, 2016

6. Campisi J: Aging, cellular senescence, and cancer. Ann Rev Physiol 75:685-705, 2013

7. Lipsitz LA, Mukai S, Hamner J, Gagnon M, Babikian V: Dynamic regulation of middle cerebral artery blood flow velocity in aging and hypertension. Stroke 31:1897-1903, 2000

8. McDougall CG, Spetzler RF, Zabramski JM, Partovi S, Hills NK, Nakaji P, et al: The Barrow Ruptured Aneurysm Trial. J Neurosurg 116:135-144, 2012

9. Molyneux A, Kerr R, Stratton I, Sandercock P, Clarke M, Shrimpton J, et al: International Subarachnoid Aneurysm Trial (ISAT) of neurosurgical clipping versus endovascular coiling in 2143 patients with ruptured intracranial aneurysms: a randomised trial. Lancet 360:1267-1274, 2002

10. Molyneux AJ, Birks J, Clarke A, Sneade M, Kerr RSC: The durability of endovascular coiling versus neurosurgical clipping of ruptured cerebral aneurysms: 18 year follow-up of the UK cohort of the International Subarachnoid Aneurysm Trial (ISAT). Lancet 385:691-697, 2015

11. Perri J, Nolan B, Goodney P, DeMartino R, Brooke B, Arya $\mathrm{S}$, et al: Factors affecting operative time and outcome of carotid endarterectomy in the Vascular Quality Initiative. J Vasc Surg 64:1177, 2016 (Abstract)

12. Silva NA, Shao B, Sylvester MJ, Eloy JA, Gandhi CD: Unruptured aneurysms in the elderly: perioperative outcomes and cost analysis of endovascular coiling and surgical clipping. Neurosurg Focus 44(5):E4, 2018

\section{Disclosures}

The authors report no conflict of interest. 